\title{
Notes on Algebraic Invariants for Non-commutative Dynamical Systems
}

\author{
Roberto Longo ${ }^{1}$ \\ Istituto Matematico G. Castelnuovo, Università di Roma, Rome, Italy
}

\begin{abstract}
We consider an algebraic invariant for non-commutative dynamical systems naturally arising as the spectrum of the modular operator associated to an invariant state, provided certain conditions of mixing type are present. This invariant turns out to be exactly the annihilator of the invariant $T$ of Connes. Further comments are included, in particular on the type of certain algebras of local observables.
\end{abstract}

\section{Preliminaries}

Following a standard terminology we say that a triple $\{\mathscr{R}, G, \alpha\}$ is $W^{*}$-system if $\mathscr{R}$ is a von Neumann algebra, $G$ is a locally compact (Hausdorff) group and $\alpha: G \rightarrow \operatorname{Aut}(\mathscr{R})$ is a representation of $G$ by $*$-automorphisms of $\mathscr{R}$ such that the map $g \in G \rightarrow \alpha_{g}(A) \in \mathscr{R}$ is ultraweakly continuous for every $A \in \mathscr{R}$.

Amply motivated both by mathematical and physical reasons, such noncommutative dynamical systems have been studied for several years by many authors, part of them being interested in particular in the determination of the algebraic type for $\mathscr{R}$ (e.g. [1-9]).

As a motivation example, let us give a direct proof, in the factor case, of a theorem of Hugenholtz [6] and Størmer [2,7] (the general proof would not be more difficult).

Theorem 1. Let $\{\mathscr{R}, G, \alpha\}$ be a $W^{*}$-system, where $\mathscr{R}$ acts on a Hilbert space $\mathscr{H}$. Let us assume the existence of a unitary representation $U$ of $G$ on $\mathscr{H}$, which implements $\alpha$, such that $U(g) \xi=\xi, g \in G$, where $\xi \in \mathscr{H}$ is cyclic for $\mathscr{R}$ and $\mathbb{C} \xi$ are the only $U$-invariant vectors. Then $\mathscr{R}$ is of type III or $\xi$ is a trace vector for $\mathscr{R}^{\prime}$.

Proof in the factor case. We can assume $\xi$ cyclic and separating for $\mathscr{R}$, otherwise considering $E \mathscr{R} E, E \equiv\left[\mathscr{R}^{\prime} \xi\right]$. Let $\omega$ be the positive functional $\omega(A)=(A \xi, \xi), A \in \mathscr{R}$, and $\sigma^{\omega}$ its modular group. We have to show that if $\mathscr{R}$ is semifinite, then $\omega$ is a trace, that is $\sigma^{\omega}$ is trivial. If $\mathscr{R}$ is semifinite there exists a one-parameter unitary group 
$V \subset \mathscr{R}$ such that

$$
\sigma_{t}^{\omega}(A)=V(t) A V(-t), \quad A \in \mathscr{R}, \quad t \in \mathbb{R} .
$$

As $\omega$ is $\alpha$-invariant, $\sigma^{\omega}$ and $\alpha$ commute, thus

$$
\begin{aligned}
\alpha_{g}(V(t)) A \alpha_{g}(V(-t)) & =\alpha_{g} \circ \sigma_{t}^{\omega} \circ \alpha_{g}^{-1}(A) \\
& =\sigma_{t}^{\omega}(A)=V(t) A V(-t), \quad A \in \mathscr{R},
\end{aligned}
$$

that is $\alpha_{g}(V(t))$ and $V(t)$ implement the same group on $\mathscr{R}$, thus

$$
\lambda(g, t) \equiv \alpha_{g}(V(t)) V(-t) \in \mathscr{R}^{\prime} \cap \mathscr{R}=\mathbb{C} I .
$$

We have

$$
\omega(V(t))=\omega\left(\alpha_{g}(V(t))\right)=\omega(\lambda(g, t) V(t))=\lambda(g, t) \omega(V(t)) .
$$

As $t \rightarrow 0, V(t)$ strongly tends to $I$ and $\omega(V(t))$ does not vanish near zero. Fixed $g \in G$, $\lambda(g, t)$ is then a character of $\mathbb{R}$ equal to 1 on a open set, therefore $\lambda(g, t)=1$ identically. It follows that $V(t)$ is a fixed element for $\alpha$. As $\xi$ is separating, the $\alpha$-fixed points are scalars, therefore $V \subset \mathbb{C} I$ and $\sigma^{\omega}$ is trivial.

The above theorem furnishes simple conditions to derive the type III property, in particular 1 must be a simple eigenvalue for $U$. However, these conditions cannot determine the invariants $S$ or $T$ of Connes [12] and a more specific analysis is needed. Assuming a strongly clustering property with respect to $\omega$ and the norm asymptotic abelianness for a dense subalgebra, Størmer [1] proved that $\operatorname{spec}(\omega)$ (the spectrum of the naturally associated modular operator) is an algebraic invariant equal to the invariant $S$ (at least in the factor case).

Here we shall deal with a much weaker assumption, which in the factor case means that the point spectrum of $U$ contains only one point and 1 is a simple eigenvalue for $U$. In such case $\operatorname{spec}(\omega)$ is still an algebraic invariant equal to the invariant $S$ for non type $\mathrm{III}_{0}$ factors and equal to the annihilator of the invariant $T$ in the general situation. The type $\mathrm{III}_{0}$ anomaly can occur, as shown in the examples.

In Appendix II we shall give a simpler and more general proof of Størmer result. This will be obtained as the combination of two propositions that may have their own interest. In such way we shall clarify the role and the localization of the effect of the asymptotic abelianness.

We shall make other remarks, for instance we present a little extension of a theorem of Driessler [3], necessary for example to derive the type $\mathrm{III}_{1}$ property for some algebras in quantum field theory, without assuming any mass gap condition.

\section{Determination of Algebraic Invariants}

Let $\{\mathscr{R}, G, \alpha\}$ be a $W^{*}$-system, $U(\mathscr{R})$ the group of the unitary operators of $\mathscr{R}$ and aut $(U(\mathscr{R}))$ its automorphism group. The representation $\alpha$ induces an action $\bar{\alpha}: G \rightarrow \operatorname{aut}(U(\mathscr{R}))$ that leaves invariant the normal subgroup $U(\mathscr{Z})$ of $U(\mathscr{R})$, where $\mathscr{Z}=\mathscr{R} \cap \mathscr{R}^{\prime}$. Thus one can naturally forms the action $\overline{\bar{\alpha}}: G \rightarrow \operatorname{aut}(U(\mathscr{R}) / U(\mathscr{Z}))$. We consider the following condition, which is somewhat of mixing type. 
Condition $M$. The only fixed point for the action $\overline{\bar{\alpha}}$ is the unity of $U(\mathscr{R}) / U(\mathscr{Z})$, i.e. $V \in U(\mathscr{R}), \alpha_{g}(V) V^{-1} \in \mathscr{Z}$, all $g \in G \Rightarrow V \in \mathscr{Z}$.

The above condition occurs in each of the following cases:

(a) $\mathscr{R}$ is a factor and $\alpha$ contains only one one-dimensional subrepresentation. Note that in this case $\alpha$ is ergodic, that is

$$
\mathscr{R}^{\alpha} \equiv\left\{A \in \mathscr{R} / \alpha_{g}(A)=A, g \in G\right\}=\mathbb{C} I,
$$

therefore any eigenoperator of norm one must be unitary. In particular we have this if $\alpha$ is weakly mixing in the sense of Doplicher and Kastler [13] with respect to a $\alpha$-invariant state $\omega \in \mathscr{R}_{*}$ :

$$
\left.m_{g}\left|\omega\left(\mathrm{A}^{*} \alpha_{g}(\mathrm{~A})\right)-\right| \omega(\mathrm{A})\right|^{2} \mid=0, \quad \mathrm{~A} \in \mathscr{R},
$$

where $m$ is the Godement mean on $G$. Indeed condition (1) is equivalent to the absence of finite-dimensional subrepresentations of $\alpha$, except the trivial onedimensional one.

Another sufficient condition for $\alpha$ to have only one eigenoperator is that $\alpha$ is strongly clustering with respect to a faithful state $\varphi \in \mathscr{R}_{*}$, in the sense that, for every $A, B \in \mathscr{R}$, there exists a sequence $g_{n} \in G$ such that

$$
\varphi\left(\alpha_{g_{n}}(A) B\right) \rightarrow \varphi(A) \varphi(B) \text { as } n \rightarrow \infty .
$$

(b) The system is asymptotically abelian in the strong topology in the sense that, for any given $A, B \in \mathscr{R}$, there exists a sequence $g_{n} \in G$ such that

$$
\left\|\left[\alpha_{g_{n}}(A), B\right] x\right\| \rightarrow 0 \quad \text { as } n \rightarrow \infty
$$

(convergence in mean suffices) for every $x$ in the underlying Hilbert space $\mathscr{H}$. To check condition $M$ let $V \in U(\mathscr{R})$ transform as $\alpha_{g}(V)=\lambda(g) V$ with $\lambda(g) \in \mathscr{Z}$. We then have

$$
\begin{aligned}
\|[V, B] x\| & =\left\|\lambda\left(g_{n}\right)[V, B] x\right\|=\left\|\left[\lambda\left(g_{n}\right) V, B\right] x\right\| \\
& =\left\|\left[\alpha_{g_{n}}(V), B\right] x\right\|_{n=\infty} \rightarrow 0, \quad x \in \mathscr{H},
\end{aligned}
$$

that entails $V \in \mathscr{R}^{\prime}$.

The condition given by formula (2) usually occurs when dealing with a local structure, namely there exists a faithful $\alpha$-invariant state $\omega \in \mathscr{R}_{*}$ strongly clustering with respect to $\alpha$ and $\alpha$ is norm asymptotically abelian on a dense subalgebra (e.g. [4]).

(c) $\mathscr{R}$ is abelian.

Lemma 1. Let $\{\mathscr{R}, G, \alpha\}$ be a $W^{*}$-system and assume that condition $M$ holds. If $C$ is the center of $G$, for every $h \in C, \alpha_{h}$ is inner iff $\alpha_{h}$ is the identity automorphism.

Proof. For a fixed $h \in C$ we have to show that if there exists $V \in \mathscr{R}$ such that $\alpha_{h}(A)$ $=V A V^{*}, A \in \mathscr{R}$, then $V \in \mathscr{R}^{\prime}$. Since $h$ commutes with every $g \in G$ we have

$$
V A V^{*}=\alpha_{h}(A)=\alpha_{g} \circ \alpha_{h} \circ \alpha_{g}^{-1}(A)=\alpha_{g}\left(V \alpha_{g}^{-1}(A) V^{*}\right)=\alpha_{g}(V) A \alpha_{g}(V)^{*} .
$$

Hence $V$ and $\alpha_{g}(V)$ implement the same group on $\mathscr{R}$, thus $\alpha_{g}(V) V^{-1} \in \mathscr{R}^{\prime} \cap \mathscr{R}$ and, by condition $M, V \in \mathscr{R}^{\prime} \cap \mathscr{R}$.

For the sake of completeness we prove the following known lemma. 
Lemma 2. Let $\{\mathscr{R}, G, \alpha\}$ be an ergodic $W^{*}$-system and $\{\mathscr{R}, H, \beta\}$ another $W^{*}$-system with $H$ abelian and $\beta$ commuting with $\alpha$. Then $\operatorname{sp}(\beta)$, the spectrum of $\beta$, is a closed subgroup of the dual of $H$.

Proof. We have to prove that if $p, q \in \operatorname{sp}(\beta)$, then $p-q \in \operatorname{sp}(\beta)$, that is the spectral subspace $\mathscr{R}(\beta, E)$ is non-trivial for every neighborhood $E$ of $p-q$. Indeed let $E_{i}$ $(i=1,2)$ be neighborhoods of $p$ and $q$ respectively such that $E_{1}-E_{2} \subset E$ and choose $0 \neq A_{i} \in \mathscr{R}\left(\beta, E_{i}\right)$. For every $g \in G$ we have $\alpha_{g}\left(A_{1}\right) A_{2}^{*} \in \mathscr{R}(\beta, E)$ ( $\alpha$ leaves invariant the $\beta$-spectral subspaces) and, for some $g \in G, \quad \alpha_{g}\left(A_{1}\right) A_{2}^{*} \neq 0$ because $F \equiv \operatorname{support}\left\{\alpha_{g}\left(A_{1}\right), g \in G\right\}$ is $\alpha$-invariant, thus $F=I$.

We now introduce an algebraic invariant for von Neumann algebras naturally appearing when dealing with condition $M$. As usual $\Delta_{\varphi}$ denotes the modular operator of a state $\varphi$.

Definition 1 . Let $\mathscr{R}$ be a von Neumann algebra. We put

$$
\operatorname{Sg}(\mathscr{R})=\bigcap_{\varphi} \operatorname{sp}\left(\Delta_{\varphi}\right)
$$

where $\varphi$ runs on the set of normal faithful states of $\mathscr{R} \operatorname{such}$ that $\operatorname{sp}\left(\Delta_{\varphi}\right)$ is a multiplicative subset of $\mathbb{R}$ [i.e. $\operatorname{sp}\left(\sigma^{\varphi}\right)$ is a group]. Such states always exist, as next proposition will show.

By its they definition $\operatorname{Sg}(\mathscr{R}) \backslash\{0\}$ is a closed subgroup of $\mathbb{R}^{+}$. We shall see that it is fully expressible in terms of the Connes invariant $T$ (the subgroup of $\mathbb{R}$, not closed in general, of all $t \in \mathbb{R}$ such that $\sigma_{t}^{\varphi}$ is inner for one, thus for every, faithful state $\varphi \in \mathscr{R}_{*}$ ). As a consequence $\mathrm{Sg}$ is not unrelated with the invariant $S$ (intersection of $\operatorname{sp}\left(\Delta_{\varphi}\right)$ for all faithful states $\varphi \in R_{*}$ ).

Proposition 1. Let $\mathscr{R}$ be a von Neumann algebra with separable predual $\mathscr{R}_{*}$. We have:

(i) There exists a faithful state $\varphi \in \mathscr{R}_{*}$ such that $\mathrm{sp}\left(\Delta_{\varphi}\right)$ is multiplicative.

(ii) $\operatorname{Sg}(\mathscr{R}) \backslash\{0\}=T(\mathscr{R})^{\perp}$ (duality between $\mathbb{R}$ and $\mathbb{R}^{+}$).

(iii) $\operatorname{Sg}(\mathscr{R}) \supset S(\mathscr{R})$ and, if $\mathscr{R}$ is a factor not of type $I I I_{0}, \operatorname{Sg}(\mathscr{R})=S(\mathscr{R})$.

The proof of this proposition will be given later. We now calculate invariants for a class of $W^{*}$-systems.

Theorem 2. Let $\{\mathscr{R}, G, \alpha\}$ be an ergodic ${ }^{2} W^{*}$-system obeying condition $M$ and $\omega \in \mathscr{R}_{*}$ an $\alpha$-invariant state. We then have

(i) $T(\mathscr{R})$ is a closed subgroup of $\mathbb{R}$ equal to the kernel of the map $t \in \mathbb{R} \rightarrow \sigma_{t}^{\omega} \in \operatorname{Aut}(\mathscr{R})$.

(ii) $\mathscr{R}$ is of type III or $\omega$ is a trace for $\mathscr{R}$.

(iii) $\operatorname{Sg}(\mathscr{R})=\operatorname{sp}\left(\Delta_{\omega}\right)$.

(iv) $T(\mathscr{R})=(\operatorname{Sg}(\mathscr{R}) \backslash\{0\})^{\perp}$ (duality between $\mathbb{R}^{+}$and $\left.\mathbb{R}\right)$.

The case $S(\mathscr{R}) \neq \operatorname{Sg}(\mathscr{R})$ can occur.

Proof. (i) Note that the support of $\omega$ is a projection of $\mathscr{R}$ which is $\alpha$-invariant, hence by ergodicity equal to $I$ and $\omega$ is faithful. We shall prove that for fixed $t \in \mathbb{R}, \sigma_{t}^{\omega}$ is inner iff $\sigma_{t}^{\omega}$ acts identically. Since $\omega$ is $\alpha$-invariant, $\sigma^{\omega}$ and $\alpha$ commute and we can form the $W^{*}$-system $\{\mathscr{R}, G \times \mathbb{R}, \alpha \circ \sigma\}$. An application of Lemma 1 yields the desired result.

2 Having condition $M$, ergodicity is equivalent to ergodicity on the center 
(ii) This is a general consequence of Theorem 1, but here it follows directly by point (i). The representation $\alpha$ leaves globally invariant the type component of $\mathscr{R}$, thus their supports are fixed points. As $\alpha$ acts ergodically, $\mathscr{R}$ is of type III or semifinite. If $\mathscr{R}$ is semifinite $T(\mathscr{R})=\mathbb{R}$, therefore by point (i) $\sigma^{\omega}$ is trivial and $\omega$ is a trace.

(iii) By Lemma $2 \operatorname{sp}\left(\sigma^{\omega}\right)$ is a group, thus $\operatorname{Sg}(\mathscr{R}) C \operatorname{sp}\left(\Delta_{\omega}\right)$. To get the reverse inclusion, we show that $\operatorname{sp}\left(\Delta_{\omega}\right) C \operatorname{sp}\left(\Delta_{\varphi}\right)$ for every faithful state $\varphi \in \mathscr{R}_{*}$ such that $\operatorname{sp}\left(\Delta_{\varphi}\right)$ is multiplicative. If $\operatorname{sp}\left(\Delta_{\varphi}\right) \subset\{0,1\}$, then $\Delta_{\varphi}=I$ and $\varphi$ is a trace; by point (ii) also $\omega$ is a trace and $\operatorname{sp}\left(\Delta_{\omega}\right)=\{1\}$. If $\operatorname{sp}\left(\Delta_{\varphi}\right)=\overline{\mathbb{R}}^{+}$the inclusion is obvious. Finally if, for some $\mu \in(0,1), \operatorname{sp}\left(\Delta_{\varphi}\right)=\left\{\mu^{n}, n \in \mathbb{Z}\right\}^{-}$, we have the following chain of implications where $t_{0}=2 \pi / \log \mu$

$$
\begin{aligned}
\operatorname{sp}\left(\Delta_{\varphi}\right)=\left\{\mu^{n}, n \in \mathbb{Z}\right\}^{-} & \Rightarrow \Delta_{\varphi}^{i t_{0}}=1 \\
& \Rightarrow \sigma_{t_{0}}^{\varphi}=1 \Rightarrow \sigma_{t_{0}}^{\varphi} \text { is inner } \Rightarrow \sigma_{t_{0}}^{\omega} \text { is inner } \\
& \Rightarrow \sigma_{t_{0}}^{\omega}=1 \Rightarrow \Delta_{\omega}^{i t_{0}}=1 \\
& \Rightarrow \operatorname{sp}\left(\Delta_{\omega}\right) \subset\left\{\mu^{n}, n \in \mathbb{Z}\right\}^{-}
\end{aligned}
$$

where we made use of the Connes cocycle Radon-Nicodym theorem and of the above point (i).

(iv) This follows easily by points (i) and (iii) or by (i) and Proposition 1.

In the examples we shall see that $\mathrm{Sg}$ may be strictly larger than $S$.

Remark 1. There can be algebraic properties that allow us to calculate $S(\mathscr{R})$ in the above theorem. For example if $\mathscr{R}$ is a factor and $\operatorname{sp}\left(\Delta_{\omega}\right) \backslash\{0\}$ is cyclic and nontrivial, then either the centralizer $\mathscr{R}^{\omega}$ is a factor and $S(\mathscr{R})=\operatorname{sp}\left(\Delta_{\omega}\right)$ or the center of $\mathscr{R}^{\omega}$ is non-atomic and $S(\mathscr{R})=\{0,1\}$.

Corollary 1. Let $\mathscr{R}$ be a factor acting on a separable Hilbert space $\mathscr{H}, \xi \in \mathscr{H}$ a cyclic vector for $\mathscr{R}$ and $U$ a unitary representation on $\mathscr{H}$ of a locally compact group $G$ such that $U(g) \mathscr{R} U(g)^{-1}=\mathscr{R}, U(g) \xi=\xi, g \in G$. Suppose that, on the orthogonal subspace of $\xi$ in $\mathscr{H}, U$ has no one-dimensional subrepresentation. Then $\operatorname{spec}(\omega) \backslash\{0\}=\operatorname{Sg}(\mathscr{R}) \backslash\{0\}$, where $\omega(A)=(A \xi, \xi), A \in \mathscr{R}$.

Proof. Let $E=\left[\mathscr{R}^{\prime} \xi\right] \in \mathscr{R}$; as $U(g) E=E U(g), g \in G$, and $\xi$ is cyclic and separating for $E \mathscr{R} E$, the $W^{*}$-system $\{E \mathscr{R} E, G, \alpha\} \alpha_{g}(E A E)=E U(g) A U(g)^{-1} E$, verifies condition $M$ and $\operatorname{spec}(\omega)=\operatorname{Sg}(E \mathscr{R} E)$ by the previous theorem. Now $\mathscr{R}$ is semifinite iff $\mathscr{R}^{\prime}$ is semifinite iff $\mathscr{R}^{\prime} E$ is semifinite $\left(\mathscr{R}^{\prime}\right.$ is isomorphic to $\left.\mathscr{R}^{\prime} E\right)$ iff $E \mathscr{R} E=\left(\mathscr{R}^{\prime} E\right)^{\prime}$ is semifinite. In such case we then have

$$
\operatorname{Sg}(\mathscr{R}) \backslash\{0\}=\operatorname{Sg}(E \mathscr{R} E) \backslash\{0\}=\{1\}
$$

by Proposition 1 . Otherwise $\mathscr{R}$ is a type III factor so that $\mathscr{R}$ is isomorphic to $E \mathscr{R} E$ and $\operatorname{Sg}(\mathscr{R})=\operatorname{Sg}(E \mathscr{R} E)$.

Corollary 2. Let $\mathfrak{U}$ be a separable unital $C^{*}$-algebra and $\varphi, \omega \in \mathfrak{U}^{*}$ factor states that are invariant and weakly mixing with respect to an action of a locally compact group (not needed to be the same for $\varphi$ and $\omega)$. If $\operatorname{spec}(\omega) \backslash\{0\} \neq \operatorname{spec}(\varphi) \backslash\{0\}$, then the GNS representation $\pi_{\varphi}$ and $\pi_{\omega}$ are disjoint. 
Proof. Being factor representations, $\pi_{\varphi}$ and $\pi_{\varphi}$ are either disjoint or quasiequivalent. The weakly mixing condition holds also in $\pi_{\omega}(\mathfrak{H})^{\prime \prime}$ and $\pi_{\varphi}(\mathfrak{U})^{\prime \prime}$, therefore if $\pi_{\omega}$ and $\pi_{\varphi}$ are quasi-equivalent, by the above corollary, we must have $\operatorname{spec}(\omega) \backslash\{0\}=\operatorname{spec}(\varphi) \backslash\{0\}$.

Lemma 3. Let $\mathscr{F}$ be a type $I_{\infty}$ factor with separable predual. For every closed subgroup $\Lambda \neq\{1\}$ of $\mathbb{R}^{+}$, there exists a faithful state $\omega \in \mathscr{F}_{*}$ such that $\operatorname{sp}\left(\Delta_{\omega}\right)=\bar{\Lambda}$.

Proof. We first construct $\omega$ with $\operatorname{sp}\left(\Delta_{\omega}\right)=\left\{\lambda^{n}, n \in \mathbb{Z}\right\}^{-}$, for a given $\lambda \in(0,1)$. $\mathscr{F}$ is isomorphic to $\mathscr{B}(\mathscr{H}), \mathscr{H}$ a separable infinite-dimensional Hilbert space. Let Tr be the trace of $\mathscr{B}(\mathscr{H}), H \in \mathscr{B}(\mathscr{H})$ a positive non-singular trace class operator such that $\operatorname{sp}(H)=\left\{\lambda^{n}, n \in \mathbb{N}\right\}^{-}$and $\omega \equiv \operatorname{Tr}(H \cdot) / \operatorname{Tr}(H)$. In a standard representation of $\mathscr{F}$ on $\mathscr{H} \otimes \mathscr{K}$, where $\mathscr{K}$ is a Hilbert space isomorphic to $\mathscr{H}$, we can identify $\Delta_{\omega}$ with $H \otimes H^{-13}$, therefore $\operatorname{sp}\left(\Delta_{\omega}\right)=\left\{\lambda^{n}, n \in \mathbb{Z}\right\}^{-}$. As to the case $\bar{\Lambda}=\overline{\mathbb{R}^{+}}$, we choose $\lambda_{1}, \lambda_{2} \in(0,1)$ with $\log \left(\lambda_{1}\right) / \log \left(\lambda_{2}\right)$ irrational and $\omega_{i}$ faithful normal states of $\mathscr{F}$ such that $\operatorname{sp}\left(\Delta_{\omega_{i}}\right)=\left\{\lambda_{i}^{n}, n \in \mathbb{Z}\right\}^{-}(i=1,2)$. Since $\mathscr{F}$ is isomorphic to $\mathscr{F} \otimes \mathscr{F}$ the state $\omega \equiv \omega_{1} \otimes \omega_{2} \in(\mathscr{F} \otimes \mathscr{F})_{*}$ is a state of $\mathscr{F}$ with $\operatorname{sp}\left(\Delta_{\omega}\right)=\overline{\mathbb{R}^{+}}$.

Proof of Proposition 1. (i) If $\mathscr{R}$ is finite the assertion is trivial. Otherwise $\mathscr{R}$ decomposes as $\mathscr{R}_{1} \oplus \mathscr{R}_{2}$ where $\mathscr{R}_{1}$ is a finite von Neumann algebra (possibly zero) and $\mathscr{R}_{2}$ is a properly infinite one. It is enough to show that $\operatorname{sp}\left(\Delta_{\varphi}\right)=\overline{\mathbb{R}^{+}}$for one normal faithful state $\varphi \in \mathscr{R}_{2 *}$, hence we can assume $\mathscr{R}$ to be properly infinite. $\mathscr{R}$ is then isomorphic to $\mathscr{R} \otimes \mathscr{F}, \mathscr{F}$ a type $I_{\infty}$ factor. By Lemma 3 there exists a faithful state $\omega \in \mathscr{F}_{*}$ with $\operatorname{sp}\left(\Delta_{\omega}\right)=\overline{\mathbb{R}^{+}}$, therefore, for any faithful state $\psi \in \mathscr{R}_{*}$, the state $\varphi \equiv \psi \otimes \omega$ of $\mathscr{R} \otimes \mathscr{F} \sim \mathscr{R}$ has $\operatorname{spectrum} \operatorname{sp}\left(\Delta_{\varphi}\right)=\overline{\mathbb{R}^{+}}$.

(ii) If $T(\mathscr{R})=\{0\}$ the assertion follows by the above point (i), hence we can assume $T(\mathscr{R})$ to be non trivial. If $\mathscr{R}$ is finite the assertion is obvious. Otherwise we shall show that, for each $t_{0} \in T(\mathscr{R}) \backslash\{0\}$, there exists a faithful normal state $\varphi \in \mathscr{R}_{*}$ with $\operatorname{sp}\left(\Delta_{\varphi}\right)=\left\{\lambda^{n}, n \in \mathbb{Z}\right\}^{-}$, where $\lambda=\exp \left(2 \pi / t_{0}\right)$. By an argument similar to that given above, we can again assume $\mathscr{R}$ to be properly infinite, thus $\mathscr{R}$ is isomorphic to $\mathscr{R} \otimes \mathscr{F}$. By [14, Theorem 1.3.2] there exists a faithful state $\psi \in \mathscr{R}_{*}$ such that $\Delta_{\varphi}^{i t_{0}}=1$. Taking a faithful state $\omega \in \mathscr{F}_{*}$, given by Lemma 3 , such that $\operatorname{sp}\left(\Delta_{\omega}\right)=\left\{\lambda^{n}, n \in \mathbb{Z}\right\}^{-}$, the state $\varphi \equiv \psi \otimes \omega \in(\mathscr{R} \otimes \mathscr{F})_{*}$ is then a state of $\mathscr{R}$ such that $\operatorname{sp}\left(\Delta_{\varphi}\right)=\left\{\lambda^{n}, n \in \mathbb{Z}\right\}^{-}$.

(iii) The inclusion is obvious from (i) and the other assertion follows from (ii) and the Theorem 3.4.1 of [14].

Remark 2. We could define $\operatorname{Sg}(\mathscr{R})$ letting $\varphi$ vary among the semi-finite faithful normal weights of $R$ with multiplicative spectra. In this case Proposition 1 is still valid with $S$ defined in terms of weights.

\section{Examples}

Condition $M$ occurs in many cases of interest, for instance in quantum field theory due to the usual presence of the strong asymptotic abelianness. The simplest example is however furnished by the Powers factors: as shown in [1] each such a

3 This follows easily identifying canonically $\mathscr{H} \otimes \mathscr{K}$ with the Hilbert-Schmidt operators $H S(\mathscr{H})$, thus $\mathscr{B}(\mathscr{H}) \otimes 1$ with $\pi(\mathscr{B}(\mathscr{H}))$, where $\pi(A) B=A B, \quad A \in \mathscr{B}(\mathscr{H}), \quad B \in H S(\mathscr{H})$, and the equality $\pi\left(\sigma_{t}^{\omega}(A)\right)=\pi\left(H^{i t} A H^{-i t}\right)$ 
factor $\mathscr{R}$ belongs to a $W^{*}$-system $\{\mathscr{R}, G, \alpha\}$, where $\alpha$ contains only the trivial onedimensional subrepresentation, as we can check easily since $\alpha$ is strongly clustering with respect to a faithful state $\omega \in \mathscr{R}_{*}$. In this way one can construct type $\mathrm{III}_{\lambda}$, $\lambda \in(0,1]$, or $\mathrm{II}_{1}$ factors, but the rich structure that one has, namely the presence of norm asymptotic abelianness for a dense $C^{*}$-subalgebra, prevents $\mathscr{R}$ from being of type III $_{0}$, thus $\operatorname{Sg}(\mathscr{R})=S(\mathscr{R})$ by Proposition 1 . We now construct examples where $\mathrm{Sg} \neq S$. All von Neumann algebras will have separable predual.

a) For every $\lambda \in(0,1)$ there exists a $W^{*}$-system $\{\mathscr{M}, H, \gamma\}$ obeying condition $M$, where $\mathscr{M}$ is a type $I I I_{0}$ factor and $\operatorname{Sg}(\mathscr{M})=\left\{\lambda^{n}, n \in \mathscr{Z}\right\}^{-}$.

Proof. We need a structure theorem of Araki [20] and Takesaki [12] of which we recall the part of interest for us. Ingredients are: a finite von Neumann algebra $\mathscr{R}_{0}$, a projection $E \in \mathscr{R}_{0}$ with $E^{\natural}=\lambda I$ and an isomorphism $\vartheta$ of $\mathscr{R}_{0}$ onto $E \mathscr{R}_{0} E$. Then one requires that the automorphism $\tilde{\vartheta}$ of $\mathscr{Z}_{0} \equiv \mathscr{R}_{0} \cap \mathscr{R}_{0}^{\prime}$ defined by $\tilde{\vartheta}(A) E=\vartheta(A)$, $A \in \mathscr{Z}_{0}$, acts ergodically on $\mathscr{Z}_{0}$ leaving invariant a state $\varphi_{0} \in \mathscr{Z}_{0 *}$. Having this, one forms a type III factor $\mathscr{R} \equiv \mathfrak{R}\left(\mathscr{R}_{0}, \vartheta\right)$ which has some of the features of a discrete cross product. $\varphi_{0}$ canonically extends to a state $\varphi \in \mathscr{R}_{*}$ with $\operatorname{sp}\left(\Delta_{\varphi}\right)=\left\{\lambda^{n}, n \in \mathbb{Z}\right\}^{-}$. Moreover an automorphism $\tau \in \operatorname{Aut}\left(\mathscr{R}_{0}\right)$ is admissible, that is it extends to a $\hat{\tau} \in \operatorname{Aut}(\mathscr{R})$ with $\varphi \circ \hat{\tau}=\varphi$, iff there exists a partial isometry $W \in \mathscr{R}_{0}$ such that

$$
\begin{aligned}
& \vartheta \circ \tau(A)=W^{*}(\tau \circ \vartheta(A)) W \\
& \tau \circ \vartheta(A)=W(\vartheta \circ \tau(A)) W^{*}, \quad A \in \mathscr{R}_{0} .
\end{aligned}
$$

Conversely any factor $\mathscr{R}$ belonging to an ergodic $W^{*}$-system $\{\mathscr{R}, G, \alpha\}$ with an $\alpha$-invariant state $\varphi \in \mathscr{R}_{*}, \operatorname{sp}\left(\Delta_{\varphi}\right)=\left\{\lambda^{n}, n \in \mathbb{Z}\right\}^{-}$, can be realized as $\mathfrak{R}\left(\mathscr{R}_{0}, \vartheta\right)$, where $\mathscr{R}_{0}=\mathscr{R}^{\varphi}$ is the centralizer of $\varphi$.

Now take $\{\mathscr{R}, G, \alpha\}$ be the above mentioned example with $\mathscr{R}$ the type III $_{\lambda}$ Powers factor, $\omega \in \mathscr{R}_{*}$ the $\alpha$-invariant state and $\left\{\mathscr{R}_{0}, G, \alpha^{0}\right\}$ the $W^{*}$-system obtained considering the restriction $\alpha^{0}$ of the representation $\alpha$ to $\mathscr{R}_{0} \equiv \mathscr{R}^{\omega}:$ as $\mathscr{R}_{0}$ is a factor the system verifies condition $M$.

Let $\{\mathscr{A}, \mathbb{Z}, \beta\}$ be a classical mixing $W^{*}$-system, that is $\mathscr{A} \neq \mathbb{C I}$ is abelian and $\beta$ is mixing with respect to a faithful state $\mu \in \mathscr{A}_{*}$.

The system $\left\{\mathscr{R}_{0} \otimes \mathscr{A}, G \times \mathbb{Z}, \alpha^{0} \otimes \beta\right\}$ is mixing with respect to $\varphi_{0} \equiv \omega \mid \mathscr{R}_{0} \otimes \mu$. Let $E \in \mathscr{R}_{0}$ and $\vartheta: \mathscr{R}_{0} \rightarrow E \mathscr{R}_{0} E$ such that $\mathscr{R}=\mathfrak{R}\left(\mathscr{R}_{0}, \vartheta\right)$. Then $\vartheta \otimes \beta_{1}$ maps $\mathscr{R}_{0} \otimes \mathscr{A}$ onto $E \otimes 1 \mathscr{R}_{0} \otimes \mathscr{A} E \otimes 1$ and $\widetilde{\vartheta \otimes \beta_{1}}=1 \otimes \beta_{1}$ acts ergodically on the center $1 \otimes \mathscr{A}$ of $\mathscr{R}_{0} \otimes \mathscr{A}$, so we can form

$$
\mathscr{M} \equiv \mathfrak{R}\left(\mathscr{R}_{0} \otimes \mathscr{A}, \vartheta \otimes \beta_{1}\right) \equiv \mathfrak{R}\left(\mathscr{M}_{0}, \vartheta_{1}\right) .
$$

The automorphisms $\alpha_{g}^{0} \otimes \beta_{n}$ are admissible for $\mathscr{M}$ : we check just the first condition in formula (3), the other one may be checked analogously. Indeed let us fix $g \in G$ and choose $W=W_{g} \in \mathscr{R}_{0}$ a partial isometry such that

$$
\vartheta \circ \alpha_{g}^{0}(A)=W^{*}\left(\alpha_{g}^{0} \circ \vartheta(A)\right) W, \quad A \in \mathscr{R}_{0}
$$

Condition (3) then holds for $\alpha_{g}^{0} \otimes \beta_{n}, n \in \mathbb{Z}$, and $W \otimes 1$ :

$$
\begin{aligned}
\vartheta \otimes \beta_{1} \circ \alpha_{g}^{0} \otimes \beta_{n}(A \otimes B) & =\vartheta \circ \alpha_{g}^{0}(A) \otimes \beta_{n+1}(B) \\
& =W^{*} \alpha_{g}^{0} \circ \vartheta(A) W \otimes \beta_{n+1}(B) \\
& =W^{*} \otimes 1 \alpha_{g}^{0} \otimes \beta_{n} \circ \vartheta \otimes \beta_{1}(A \otimes B) W \otimes 1 .
\end{aligned}
$$


Therefore the automorphisms $\alpha_{g}^{0} \otimes \beta_{n}, g \in G, n \in \mathbb{Z}$, extend to automorphisms $\overline{\alpha_{g}^{0} \otimes \beta_{n}}$ of $\mathscr{M}$ commuting with $\sigma^{\varphi}$, the modular group of the extension of $\varphi_{0}$. The factor $\mathscr{M}$ is of type $\mathrm{III}_{0}$ (because the center of $\mathscr{M}^{\varphi}, 1 \otimes \mathscr{A}$, is infinite-dimensional), $\operatorname{sp}\left(\Delta_{\varphi}\right)$ $=\left\{\lambda^{n}, n \in \mathbb{Z}\right\}^{-}$and the $W^{*}$-system $\{\mathscr{M}, H, \gamma\}$, where $H=G \times \mathbb{Z} \times \mathbb{R}$ and $\gamma=\widehat{\alpha^{0} \otimes \beta} \otimes \sigma^{\varphi}$, verifies condition $M$. To check the last assertion we note that if $V \in \mathscr{M}$ is a unitary eigenoperator for $\gamma$, then $V \in \mathscr{M}^{\varphi}$ (see [12] or the end of the proof of Theorem 3). Therefore $V$ is a unitary eigenoperator for $\alpha^{0} \otimes \beta$, thus $V \in \mathbb{C} I$ since $\alpha^{0} \otimes \beta$ verifies condition $M$ by construction. Finally by Theorem 2 we have $\operatorname{Sg}(\mathscr{M})$ $=\operatorname{sp}\left(\Delta_{\varphi}\right)=\left\{\lambda^{n}, \mathrm{n} \in \mathbb{Z}\right\}^{-}$.

$\beta)$ In the above example we can require that $\gamma$ is weakly mixing with respect to an invariant state $\varphi \in \mathscr{M}_{*}$.

Proof. It is enough to modify the above example to have $G$ abelian, thus $H$ abelian [see point (a) of Sect. 2]. We get this changing the $W^{*}$-system $\{\mathscr{R}, G, \alpha\}$ with the one considered in [15] where $G=\mathbb{R}$.

Remark 3. Weakly mixing conditions do not suffice to have $\mathrm{Sg}=S$ and it should be interesting to give other conditions to have this. Norm asymptotic abelianness on a dense subset suffices, but this condition is a very strong property of the representation. More interesting would be conditions on the state. See however Appendix II.

Up to now we have seen that almost every combination between $S$ and $\mathrm{Sg}$ allowed by Proposition 1 can actually occur in Theorem 2. For a general von Neumann algebra $\mathscr{R}$, we can also have $S(\mathscr{R})=\operatorname{Sg}(\mathscr{R})=\{0,1\}$, namely when $T(\mathscr{R}) \neq \mathbb{R}$ and $\overline{T(\mathscr{R})}=\mathbb{R}$, but this cannot occur in Theorem 2 because $T(\mathscr{R})$ is closed. The only unexamined possibility is $S(\mathscr{R})=\{0,1\}$ together with $\operatorname{Sg}(\mathscr{R})=\overline{\mathbb{R}^{+}}$. We do not have examples of this sort when condition $M$ occur. However, in view of possible future investigations, we explicitely state in the Appendix I an example of type $\mathrm{III}_{0}$ factor with $\mathrm{Sg}=\overline{\mathbb{R}^{+}}$, see also Connes [14], Hamachi et al. [22] and Krieger [23].

\section{The Case of a Semigroup Action}

In von Neumann algebras of physical provenience one also meets more general objects than $W^{*}$-systems $\{\mathscr{R}, G, \alpha\}: G$ is only required to be a semigroup and $\alpha$ is an action of $G$ by injective endomorphisms of $\mathscr{R}$. The above used techniques are no longer useful because $\alpha$ need not to commute with the modular group, but one is amply compensated having a spectral condition for $\alpha$. An illustration of this fact is given by the following extension of a theorem of Driessler. Really our statement is little more general, than the original one [3], not using the cluster property of the vacuum, but it is sufficient to get the type III $_{1}$ property for the algebras associated to wedge regions (e.g. defined by $x_{1} \geqq\left|x_{0}\right|$ ) in quantum field theory, without assuming any mass gap condition, and to the forward light cone (defined by $\left.\mathrm{x}_{0} \geqq|\mathbf{x}|\right)$ in free massless fields.

Theorem 3. Let $\mathscr{R}$ be a von Neumann algebra acting on a Hilbert space $\mathscr{H}, \xi \in \mathscr{H}$ a separating unit vector for $\mathscr{R}, G$ a locally compact abelian group with dual $\Gamma$ and $U$ a 
unitary representation of $G$ on $\mathscr{H}$ such that $U(g) \xi=\xi, g \in G$, and $\mathbb{C} \xi$ are the only $U$-invariant vectors. Suppose that there exist subsets $G_{+} \subset G$ and $\Gamma_{+} \subset \Gamma$ such that:

(a) $G_{+} \cup\left(-G_{+}\right)=G$ and $U(g) \mathscr{R} U(g)^{-1} \subset \mathscr{R}, \quad g \in G_{+}$

(b) $\Gamma_{+} \cap\left(-\Gamma_{+}\right)=\{0\}$ and $\operatorname{sp}(U) \subset \Gamma_{+}$.

Then $\mathscr{R}$ is a type $I I I_{1}$ factor or $\mathscr{R}=\mathbb{C} I$.

Proof. Let $E$ be a selfadjoint projection of the centralizer $\mathscr{R}^{\omega}$ of the state $\omega(A)$ $=(A \xi, \xi), A \in \mathscr{R}$. For every $g \in G_{+}, U(g) E U(-g) \in \mathscr{R}$, therefore

$$
\begin{aligned}
F(g) & \equiv(E U(g) E \xi, \xi)=(E U(g) E U(-g) \xi, \xi) \\
& =(U(g) E U(-g) E \xi, \xi)=(E U(-g) E \xi, \xi)
\end{aligned}
$$

that is $F(g)=F(-g), g \in G_{+}$, and, by property (a), $F(g)=F(-g)$ for every $g \in G$. Now $F$ is a positive-definite function, hence it is the Fourier transform of a measure $\hat{F}$ on $\Gamma$ that verifies $\hat{F}(p)=\hat{F}(-p), p \in \Gamma$. By assumption (b) we have support $(\hat{F}) \subset \Gamma_{+}$, thus support $(\hat{F}) \subset \Gamma_{+} \cap\left(-\Gamma_{+}\right)=\{0\}$ and this implies that $F$ is constant. In particular $F(g)=F(0), g \in G$, therefore

$$
(U(g) E \xi, E \xi)=(E \xi, E \xi)=\|E \xi\|^{2}=\|U(g) E \xi\|\|E \xi\|, \quad g \in G .
$$

By the limit case of the Schwartz inequality we then have $U(g) E \xi=\chi(g) E \xi$, when $\chi(g)$ is a complex number, therefore

$$
F(g)=(U(g) E \xi, E \xi)=\chi(g)(E \xi, E \xi)=(E \xi, E \xi) .
$$

Hence, if $E \xi \neq 0$, we have $\chi(g)=1$ and $U(g) E \xi=E \xi$, that is $E \xi$ is a $U$-invariant vector, thus $E \xi=\xi$ by assumption. As $\xi$ is separating we then have $E=0$ or $E=I$, thus $\mathscr{R}^{\omega}=\mathbb{C} I$. Now the triviality of $\mathscr{R}^{\omega}$ entails firstly that $\mathscr{R}$ is a factor, because $\mathscr{R} \cap \mathscr{R}^{\prime} \subset \mathscr{R}^{\omega}$, and secondly $\mathscr{R}$ is of type III $_{1}$ (if non-trivial): this can be viewed in a lot of manners. For example we can see by Lemma 2 that $\operatorname{sp}\left(\sigma^{\omega}\right)$ is a closed group. If $\operatorname{sp}\left(\sigma^{\omega}\right)=\{0\}$, then $\omega$ is a trace and $\mathscr{R}=\mathscr{R}^{\omega}=\mathbb{C} I$. If $\operatorname{sp}\left(\sigma^{\omega}\right)=\mathbb{R}, \mathscr{R}$ is of type $\mathrm{III}_{1}$ due to the equality $S(\mathscr{R}) \backslash\{0\}=\Gamma\left(\sigma^{\omega}\right)$ [14, Theorem 3.2.1]. The remaining case $\operatorname{sp}\left(\sigma^{\omega}\right)$ cyclic and non-trivial cannot occur. Otherwise there should exist $\lambda \in(0,1)$ and $V \in \mathscr{R}$ of norm 1 such that $\sigma_{t}^{\omega}(V)=\lambda^{i t} V, t \in \mathbb{R}$. By the KMS property one easily derives [12]:

$$
\omega(V A)=\lambda \omega(A V), \quad A \in \mathscr{R} .
$$

As $V^{*} V, V V^{*} \in \mathscr{R}^{\omega}=\mathbb{C} I, V$ is unitary and

$$
1=\omega\left(V V^{*}\right)=\lambda \omega\left(V^{*} V\right)=\lambda
$$

that is a contradiction.

We now mention two examples where the above theorem can be applied. Other examples can be easily shown. The algebras in the following corollary are those ones associated to regions of the Minkowski space in algebraic quantum field theory (see e.g. [17, Chap. 23]). 
Corollary 3. The following von Neumann algebras are factors of type $I I I_{1}$.

(i) Algebras associated to wedge regions.

(ii) Algebras associated to light cones in the free scalar field of mass zero ${ }^{4}$.

Proof. In both cases one applies the previous theorem with $U$ a one-parameter group of light-like translations and $\xi$ the vacuum vector. $\xi$ is cyclic and separating owing to the Reeh-Schlieder theorem and the commutation of space-like separated observables as well as, in free massless fields, of observables localized in the forward light cone with those ones localized in the past light cone.

In a Wrightman theory, the results contained in Corollary 3 follow from the knowledge of the modular operator associated to the vacuum vector; see $[11,10]$ and [16] respectively.

Remark 4. If the vacuum vector is not separating for the von Neumann algebra $\mathscr{R}$ associated to the forward light cone, Theorem 3 , applied to $\mathscr{R}^{\prime}$, entails that either $\mathscr{R}^{\prime} \neq \mathbb{C} I$ and $\mathscr{R}$ is again of type $\mathrm{III}_{1}$ or $\mathscr{R}^{\prime}=\mathbb{C} I$ and $\mathscr{R}=\mathscr{B}(\mathscr{H})$. The last case occurs when dealing with a mass gap condition [18].

\section{Appendix I}

The purpose of this appendix is to give explicitly an example of type $\mathrm{III}_{0}$ factor with $\mathrm{Sg}=\overline{\mathrm{R}^{+}}$. This can be easily done making use of Takesaki duality theory [19].

$\gamma$ ) In the following construction $M$ is a factor of type $I I I_{0}$ and $\operatorname{Sg}(\mathscr{M})=\overline{\mathbb{R}^{+}}$.

Construction. By Proposition 1, we must exhibit a factor $M$ such that $S(\mathscr{M})=\{0,1\}$ and $T(\mathscr{M})=\{0\}$. The construction is somewhat similar to that given in example $\alpha$ ). Let $\mathscr{R}$ be a type $\mathrm{III}_{1}$ factor, $\varphi \in \mathscr{R}_{*}$ a faithful state and $\mathscr{R}_{0} \equiv W^{*}\left(\mathscr{R}, \mathbb{R}, \sigma^{\varphi}\right)$ the cross product of $\mathscr{R}$ by $\sigma^{\varphi}$. $\mathscr{R}_{0}$ is a type $\mathrm{II}_{\infty}$ factor and the dual action $\vartheta$ of $\sigma^{\varphi}$ on $\mathscr{R}_{0}$ verifies $\tau \circ \vartheta_{t}=e^{-t} \tau, t \in \mathbb{R}$, where $\tau$ is the trace of $\mathscr{R}_{0}[19$, Theorems 8.2, 9.7]. Let $\{\mathscr{A}, \mathbb{R}, \alpha\}$ be a $W^{*}$-system with $\mathscr{A}$ abelian and $\alpha$ mixing with respect to an $\alpha$-invariant state $\mu \in \mathscr{A}_{*}$. We consider the action of $\mathbb{R}$ on $\mathscr{M}_{0} \equiv \mathscr{R}_{0} \otimes \mathscr{A}$ given by

$$
\bar{\vartheta}: t \in \mathbb{R} \rightarrow \vartheta_{t} \otimes \alpha_{t} \in \operatorname{Aut}\left(\mathscr{M}_{0}\right) \text {. }
$$

$\mathscr{M}_{0}$ is a semifinite von Neumann algebra, $\bar{\tau} \equiv \tau \otimes \mu$ is a faithful normal semifinite trace on $\mathscr{M}_{0}$ and $\bar{\vartheta}$ diminishes $\bar{\tau}$ according to

$$
\bar{\tau} \circ \bar{\vartheta}_{t}=e^{-t} \bar{\tau}, \quad t \in \mathbb{R} \text {. }
$$

The theory of Takesaki entails then that $\mathscr{M} \equiv W^{*}\left(\mathscr{M}_{0}, \mathbb{R}, \bar{\vartheta}\right)$ is a factor of type $\operatorname{III}_{0}$ with trivial invariant $T$. Indeed the restriction of $\bar{\vartheta}$ to $\mathscr{M} \cap \mathscr{M}^{\prime}$ acts ergodically, being equal to $\alpha$, thus $\mathscr{M}$ is a factor [19, Theorem 8.5]. Moreover $S(\mathscr{M})=\{0,1\}$ and $T(\mathscr{M})=\{0\}$ because $\alpha$ is not periodic and the point spectrum of $\alpha$ contains only one element [19, Theorem 9.6 and Theorem 9.4].

\section{Appendix II}

The result of Størmer mentioned in the preliminaries can be easily derived following in part an argument of Herman [20]. We shall say that a $W^{*}$-system

4 Owing to the results of Buchholz [16], the same applies to any theory of local algebras in which there is a massless scalar one-particle state generating a complete set of asymptotic states

5 The existence of such factors follows as a special case of Theorem 3.4.4 of [14]; we thank Prof. A. Connes for pointing out this fact to us 
$\{\mathscr{R}, G, \alpha\}$ verifies the three-fold factorization property (cf. [20]) with respect to the von Neumann algebra $A \in \mathscr{R}$ if for every projection $0 \neq E \in A$ we have:

$$
A \in \mathscr{R}, \quad E \alpha_{g}(A) E=0, \text { all } g \in G \Rightarrow A=0 .
$$

In the following $S^{\prime}(\mathscr{R}) \equiv \cap \operatorname{spec}(\omega)$, where $\omega$ runs on the normal states of $\mathscr{R}$, and $\Gamma(\beta)$ is the Connes spectrum of $\beta$ (see [1], [14] resp.).

Proposition 2. Let $\{\mathscr{R}, G, \alpha\}$ and $\{\mathscr{R}, H, \beta\}$ be $W^{*}$-systems, where $H$ is abelian and $\alpha$ commutes with $\beta$. If $\{\mathscr{R}, G, \alpha\}$ verifies the three-fold factorization property with respect to the center of $\mathscr{R}^{\beta}$, then $\Gamma(\beta)=\operatorname{sp}(\beta)$.

Proof. Given $p \in \operatorname{sp}(\beta)$, we have to show that $\mathscr{R}\left(\beta^{E}, F\right) \neq\{0\}$ for every projection $E \neq 0$ of the center of $\mathscr{R}^{\beta}$ and every neighborhood $F$ of $p$. Indeed let $0 \neq A \in \mathscr{R}(\beta, F)$. Since $\alpha$ commutes with $\beta$ we have $\alpha_{g}(A) \in \mathscr{R}(\beta, F)$, thus $E \alpha_{g}(A) E \in \mathscr{R}\left(\beta^{E}, F\right), g \in G$. Owing to the three-fold factorization property we can choose $g \in G$ such that $E \alpha_{g}(A) E \neq 0$.

Proposition 3. Let $\mathscr{R}$ be a von Neumann algebra and $\omega$ a faithful normal state of $\mathscr{R}$. Then $\Gamma\left(\sigma^{\omega}\right)=S^{\prime}(\mathscr{R}) \backslash\{0\}$ (with the natural duality of $\mathbb{R}$ with $\mathbb{R}^{+}$). In particular $S^{\prime}(\mathscr{R}) \backslash\{0\}$ is a closed subgroup of $\mathbb{R}^{+}$.

Proof. By the KMS condition, if $E$ is a projection of $\mathscr{R}^{\omega}$, then $\sigma^{\omega E}$ is the modular group of $\omega \mid E \mathscr{R} E$, whence $S^{\prime}(\mathscr{R}) \backslash\{0\} \subset \Gamma\left(\sigma^{\omega}\right)$ and we have to show that $\Gamma\left(\sigma^{\omega}\right) \subset S^{\prime}(\mathscr{R})$. If there exists a semifinite projection $0 \neq G \in \mathscr{R} \cap \mathscr{R}^{\prime} \subset \mathscr{R}^{\omega}$ and $\tau$ is a semifinite normal faithful trace on $\mathscr{R} G$, then, by the Connes cocycle Radon-Nikodym theorem, we have $\Gamma\left(\sigma^{\omega}\right) \subset \Gamma\left(\sigma^{\omega^{G}}\right)=\Gamma\left(\sigma^{\tau}\right)=\{1\}$; therefore we can assume $\mathscr{R}$ to be of type III. By definition we have $S^{\prime}(\mathscr{R})=\cap S(E \mathscr{R} E)$, where $E \neq 0$ runs among the projections of $\mathscr{R}$. Since $\mathscr{R}$ is purely infinite, every projection $E \in \mathscr{R}$ is equivalent to its central cover $c(E)$, thus $\mathscr{R}$ is isomorphic to $\mathscr{R} c(E)$ and

$$
S^{\prime}(\mathscr{R})=\bigcap_{G} S(\mathscr{R} G), \quad G \neq 0 \quad \text { projection of } \mathscr{R} \cap \mathscr{R}^{\prime} .
$$

Since $\Gamma\left(\sigma^{\omega}\right)=\Gamma\left(\sigma^{\varphi}\right)$ for every faithful state $\varphi \in \mathscr{R}_{*}$, we have

$$
\Gamma\left(\sigma^{\omega}\right)=\bigcap_{\varphi} \Gamma\left(\sigma^{\varphi}\right) \subset \bigcap_{\varphi} \operatorname{spec}(\varphi)=S(\mathscr{R}) .
$$

If we apply this inclution to the state $\omega \mid \mathscr{R} G$ and we then make the intersection for every projection $0 \neq G \in \mathscr{R} \cap \mathscr{R}^{\prime}$ we get

$$
\Gamma\left(\sigma^{\omega}\right)=\bigcap_{G} \Gamma\left(\sigma^{\omega^{G}}\right) \subset \bigcap_{G} S(\mathscr{R} G)=S^{\prime}(\mathscr{R}) .
$$

Theorem 4. Let $\{\mathscr{R}, G, \alpha\}$ be a $W^{*}$-system and $\omega \in \mathscr{R}_{*}$ a faithful $\alpha$-invariant state. If the three-fold factorization property holds for $\{\mathscr{R}, G, \alpha\}$ with respect to the center of $\mathscr{R}^{\omega}$, then $\operatorname{spec}(\omega)=S^{\prime}(\mathscr{R})$.

Proof. As $\omega$ is $\alpha$-invariant, $\sigma^{\omega}$ commutes with $\alpha$. By Propositions 2 and 3 we then have

$$
\operatorname{spec}(\omega) \backslash\{0\}=\operatorname{sp}\left(\sigma^{\omega}\right)=\Gamma\left(\sigma^{\omega}\right)=S^{\prime}(\mathscr{R}) \backslash\{0\} .
$$

Since $0 \notin S^{\prime}(\mathscr{R})$ iff $S^{\prime}(\mathscr{R})=\{1\}$, we have $\operatorname{spec}(\omega)=S^{\prime}(\mathscr{R})$.

Corollary 5. Let $\{\mathscr{R}, G, \alpha\}$ be a $W^{*}$-system and $\omega \in \mathscr{R}_{*}$ an $\alpha$-invariant state whose 
(i) The system $\{\mathscr{R}, G, \alpha\}$ is asymptotically abelian in the strong topology and $\omega$ is $\alpha$-strongly clustering with respect to the same sequence $g_{n} \in G$.

(ii) $\alpha$ is centrally ergodic and the center of $\mathscr{R}^{\omega} \equiv\{A \in \mathscr{R} / \omega(A B)=\omega(B A), B \in \mathscr{R}\}$ is equal to the center of $\mathscr{R}$.

Then $\operatorname{spec}(\omega)=S^{\prime}(\mathscr{R})$.

Proof. Since $c(F)=I$, we have $S^{\prime}(\mathscr{R})=S^{\prime}(F \mathscr{R} F)$. In fact $\mathscr{R}$ has a semifinite direct summand iff the same is true for $F \mathscr{R} F$, in which case $S^{\prime}(\mathscr{R})=S^{\prime}(F \mathscr{R} F)=\{1\}$. Otherwise $\mathscr{R}$ is of type III, $F$ is equivalent to $I, \mathscr{R}$ is isomorphic to $F \mathscr{R} F$ and again $S^{\prime}(\mathscr{R})=S^{\prime}(F \mathscr{R} F)$. The corollary is then proved if we show that the $W^{*}$-system $\left\{F \mathscr{R} F, G, \alpha^{F}\right\}$ verifies the assumptions of Theorem 4 .

(i) Let $E<F$ be a projection of $\mathscr{R}$ and $A=F A F \in \mathscr{R}$. If $E \alpha_{g}(A) E=0$ for every $g \in G$, then

$$
\begin{aligned}
& E \alpha_{g}\left(A^{*} A\right) E+E \alpha_{g}\left(A^{*}\right)\left[E, \alpha_{g}(A)\right] E=E \alpha_{g}\left(A^{*}\right) E \alpha_{g}(A) E=0 \\
& \omega\left(E \alpha_{g_{n}}\left(A^{*} A\right) E\right)+\omega\left(E \alpha_{g_{n}}\left(A^{*}\right)\left[E, \alpha_{g_{n}}(A)\right] E\right)=0 .
\end{aligned}
$$

As $n \rightarrow \infty$ the left member of the last equality converges to $\omega(E) \omega\left(A^{*} A\right)$, that must be zero. Since $\omega \mid F \mathscr{R} F$ is faithful, we have $A=0$.

(ii) It is easily seen that $F \mathscr{R}^{\omega} F$ is the centralizer of $\omega \mid F \mathscr{R} F$, thus we can assume that $\omega$ is faithful. The result then follows by Theorem 4 , because the three-fold factorization property with respect to the center is really a two-fold property and it is entailed by the ergodicity on the center.

Note that in Theorem 4 one can deal with a relatively $\alpha$-invariant faithful semifinite normal weight $\omega$. In the point (i) of Corollary 4 one can require that the asymptotic abelianness and the cluster property are taken in mean; however some cluster assumption is necessary, as shown by the simple example given by the $W^{*}$-system $\{\mathscr{R} \oplus \mathscr{A}, G, \alpha \oplus j\}$, where $\{\mathscr{R}, G, \alpha\}$ is a $W^{*}$-system like in the above point (i) and $j$ is the trivial action of $G$ on an abelian von Neumann algebra $\mathscr{A}$; nevertheless, removing the cluster condition and assuming e.g. that $\omega$ is faithful, one has $\operatorname{spec}(\omega)=S(\mathscr{R})[5]$. Finally, in connection with Remark 3, we note that the three-fold factorization property can be derived by a property of the faithful state $\omega$, namely $\omega\left(E \alpha_{h}\left(A^{*}\right) E \alpha_{g}(A)\right)$ must approach $\omega(E) \omega\left(A^{*} A\right)$ in some sense.

Acknowledgements. The author is deeply indebted to S. Doplicher for many instructive and useful comments. Warm thanks are also due to C. D'Antoni for several discussions.

\section{References}

1. Størmer, E.: On the structure theory of factors. In : $C^{*}$-algebras and their applications to statistical mechanics and quantum field theory. Proceedings of the International School of Physics "Enrico Fermi", Varenna 1973. (ed. D. Kastler) North-Holland 1976

2. Størmer, E.: Automorphisms and invariant states of operator algebras. Acta Math. 127, 1-9 (1971)

3. Driessler, W.: Comments on lightlike translations and applications in relativistic quantum field theory. Commun. Math. Phys. 44, 133-141 (1975)

4. Driessler, W.: On the type of local algebras in quantum field theory. Commun. Math. Phys. 43, 295-297 (1977)

5. Araki, H.: Remarks on the spectra of modular operators of von Neumann algebras. Commun. Math. Phys. 28, 267-277 (1972) 
6. Hugenholtz, N.M.: On the factor type of equilibrium states in quantum statistical mechanics. Commun. Math. Phys. 6, 189-193 (1967)

7. Størmer, E.: Types of von Neumann algebras associated with extremal invariant states. Commun. Math. Phys. 6, 194-204 (1967)

8. Testard, D.: Asymptotic ratio set of von Neumann algebras generated by temperature states in statistical mechanics. Rep. Math. Phys. 12, 115-118 (1977)

9. Testard, D.: Some properties of the quasilocal observables in statistical mechanics and quantum field theory. Conference on "Algebras of operators and their applications in mathematical physics". Marseille, June 1977

10. Kastler, D., Wichman, E.: Unpublished

11. Bisognano, J.J., Wichman, E.H.: On the duality condition for quantum fields. J. Math. Phys. 17, 303-321 (1976). See also Rigotti, C.: On the essential duality condition for hermitian scalar fields. Conference on "Algebras of operators and their applications in mathematical physics. Marseille, June 1977

12. Takesaki, M.: The structure of a von Neumann algebra with a homogeneous periodic state. Acta Math. 131, 79-121 (1973)

13. Doplicher, S., Kastler, D. : Ergodic states in a non-commutative ergodic theory. Commun. Math. Phys. 7, 1-20 (1968)

14. Connes, A.: Une classification des facteurs de type III. Ann. Ecole Norm. Sup. 6, 133-252 (1973)

15. Emch, G.: Generalized $K$-flows. Commun. Math. Phys. 49, 191-215 (1976)

16. Buchholz, D.: On the structure of local quantum fields with non-trivial interaction. Preprint

17. Bogolubov, N.N., Logunov, A.A., Todorov, I.T.: Introduction to axiomatic quantum field theory. Benjamin 1975

18. Sadowski, P., Woronowicz, S.L.: Total sets in quantum field theory. Rep. Math. Phys. 2, 113-120 (1971)

19. Takesaki, M. : Duality for crossed product and the structure of von Neumann algebras of type III. Acta Math. 131, 244-310 (1973)

20. Herman, R.H.: Spectra of automorphism groups of operator algebras. Duke Math. J. 41, 667-673 (1974)

21. Araki, H.: Structure of some von Neumann algebras with isolated discrete modular spectrum. Publ. RIMS, Kyoto Univ. 9, 1-14 (1973)

22. Hamachi, T., Oka, Y., Osikawa, M. : Flow associated with ergodic non-singular transformation groups. Publ. RIMS, 11, 31-50 (1975)

23. Krieger, W.: On ergodic flows and the isomorphism of factors. Math. Ann. 223, 19-70 (1976)

Communicated by H. Araki

Received October 23, 1978; in revised form June 19, 1979 
